\title{
Analytic approaches to understanding future Defence workforce needs
}

\author{
C. Rowe, A. Crockett, S. Rohan-Jones, S. Aljoofri, N. Tay, A. Coutts, C. Bishop, A. Pay, \\ W. Sim, V. Date and J. Boyce \\ Department of Defence, Canberra, Australian Capital Territory \\ Email: cayt.rowe@dst.defence.gov.au
}

\begin{abstract}
A campaign of analysis has been conducted to help workforce planners to understand future Defence workforce requirements and design coherent, achievable and targeted workforce growth options for the Australian Defence Force (ADF). This paper reviews the wide range of analytical approaches that were employed to underpin the potential growth options, which will generate a robust, resilient and effective Defence workforce over the next 20 years.
\end{abstract}

In 2020, Australian Department of Defence released the Defence Strategic Update, accompanied by a new Force Structure Plan which detailed the government's intentions for capability investments. This 2020 Force Structure Plan identified that the Defence workforce needs to grow in order to support a larger number of morecomplex capabilities, including to build capacity in cyber, intelligence, electronic warfare and space. An analysis campaign to review the long-term workforce requirements for Defence became the focus of the 20202021 Defence Capability Assessment Program, which is the primary force design process for the ADF. This analysis campaign brought together two distinct disciplines of capability-based planning and workforce planning. Capability-based planning focusses on maximising the "capability" of the force, being the ability to achieve an effect, and testing the effectiveness of these capabilities within the context of scenarios. However, understanding the strengths and limitations of workforce capability is much more challenging than the traditional assessment of weapon systems, and analysis methods from human resource management are also needed to ensure an achievable workforce plan.

Five complementary analytical approaches were employed, drawing on expertise in Defence People Group, Defence Science and Technology Group, and the Force Design Division of the ADF Headquarters. Based on a series of wargames, operational demand modelling provided a detailed estimation of the number and types of people Defence will need to meet a future warfighting scenario. A Bayesian network model captures how the constituent parts of Defence contribute in a warfighting context, and this model was used to quantify the expected benefit from proposed workforce investments. The supporting and enabling functions of Defence were considered through both a prioritisation of proposed investments and mapping how the entire organisation supports Defence capability.

Whereas the above methods focussed on future demand for workforce to deliver capability, supply modelling focussed on the ability to grow and train that workforce. The workforce growth model provides realistic estimations of achievable growth rates to inform both the likely limit on how quickly Defence can grow overall, and the ability to generate specific skill sets in different locations. It also provides guidance for how many recruits should be targeted to achieve the desired number of trained personnel, and this in turn informs the required investment in supporting functions such as facilities and recruitment.

The campaign of analysis to understand future Defence workforce requirements created new connections between different analysis methods. This strengthened each individual approach and enabled the design of coherent, achievable and targeted workforce growth options for Defence. The analytical campaign described in this paper has provided much-needed detail to underpin how Defence will reshape and reskill its workforce in response to emerging requirements.

Keywords: Force design, capability development, decision making, personnel management workforce planning 


\section{INTRODUCTION}

This paper reviews the analytical approaches used to help the Australian Department of Defence (Defence) to understand future workforce requirements and design coherent, achievable and targeted workforce growth options for the Australian Defence Force (ADF) service members and the Australian Public Service (APS) civilian personnel. The goal of this analysis was to enable rational decision-making under extreme complexity and generate a robust, resilient and effective Defence workforce over the next 20 years.

The force design process is about planning for what the Australian Government will invest in to ensure that Defence capabilities are suitable for current and emerging threats. Choosing the best balance of military capabilities, for example choosing between investment in submarines and in cyber capability, is complex and challenging. It needs to consider the government's objectives for Defence, as well as budgetary resources and analysis of likely future scenarios. Traditionally, force design focusses on capital investments in warfighting assets such as submarines, jets and missiles. Analysis methods such as wargames and simulation are employed to understand the capability benefits that these investments may generate in future warfighting scenarios. These methods are particularly helpful when informing the choice of which type of missile or type of tank will be most effective in the circumstances they might face. However, these analytical tools tend to emphasise the "pointy end" of warfighting capability at the expense of enabling capabilities.

The 2020 Force Structure Plan (FSP20) recognised the changing demands on the Defence workforce. " $A$ larger number of more-complex capabilities requires growth in the size of the workforce.... Defence must also reshape and reskill its workforce to transition to new platforms and build capacity in emerging capabilities such as cyber, intelligence, electronic warfare and space." (Dept of Defence, 2020, p. 103) Reviewing the long-term workforce needs became the focus of the 2020-2021 Defence Capability Assessment Program (DCAP), which is the primary force design process for the $\mathrm{ADF}$.

\section{BACKGROUND}

This analysis campaign brought together two distinct disciplines of capability-based planning and workforce planning. Capability-Based Planning (CBP) is one approach to Defence capability development and a form of long-term Defence Planning. CBP focusses on maximising the "capability" of the force, being the ability to achieve an effect, and testing the effectiveness of these capabilities within the context of scenarios. However, understanding the strengths and limitations of workforce capability is much more challenging than the traditional assessment of weapon systems, and analysis methods from human resource management are also needed to ensure an achievable workforce plan.

At the simplest level, the Defence workforce can be described in terms of the number of ADF and APS personnel employed and, in the past, it's been largely assumed that changes to duties and skills within the workforce can be made relatively quickly when compared to the 10 to 20 years required to deliver a new warship. However, FSP20 made it clear that the looming workforce challenges cannot be solved by simply moving people from one work area to another and a wholesale review of Defence workforce needs was required.

Since 2016, the ADF Total Workforce System has been reviewed and modernised to improve recruitment, retention and flexibility. FSP20 also highlighted the need to enlarge and update training facilities and other infrastructure such as accommodation, garrison services and information, communications and technology (ICT) support (Dept of Defence, 2020, p. 107). This level of investment requires a clear articulation of how many and what types of people Defence needs to grow; it also needs details about where they will be based and what support infrastructure they will need.

\section{ANALYTIC APPROACHES TO UNDERSTANDING FUTURE DEFENCE WORKFORCE NEEDS}

The analysis that supported the 2020-2021 Defence review of future workforce needs was a strong collaboration across different parts of the Department, including the Defence Science and Technology Group (DSTG), Defence People Group (DPG), and Force Design Division (FDD) within the Australian Defence Force Headquarters. It also made use of Project Global Endeavour and their experience in modelling of workforce demands for planning current operations. 
These different groups employed different analytic approaches to inform planning of the Defence Workforce for the next twenty years. The approaches can be divided into three categories:

1. Workforce requirements to meet future warfighting scenarios (sections 3.1 and 3.2)

2. Evolution of existing workforce to meet Defence capability outcomes (sections 3.3 and 3.4)

3. Longitudinal workforce growth achievability and supply models (section 3.5)

\subsection{Estimating Operational Demand for Workforce}

The first two approaches build on a typical force design analysis, where Defence capabilities are assessed against a range of possible warfighting scenarios. In DCAP2020-21, the analysis focussed on a set of concurrent vignettes that comprised a single Theatre Problem set in 2029. Detailed analysis of this Theatre Problem enabled the challenges around support functions such as logistics and command and control (C2) to be explored against a specific, well-defined problem.

This contrasts with the more typical approach to force design, which is to conduct a broad brush overview of a number of completely independent scenarios, allowing for more complete coverage of the range of tasks that the ADF may be required to undertake. However, that broader scope means, firstly, that there is rarely enough time to explore the details of the enabling functions for each scenario, and secondly that these scenarios don't address concurrent demands on Defence and other government agencies that may be expected in a time of war.

A series of seminar wargames were conducted to explore the major lines of operation (LOOs) within the Theatre Problem. These were operational-level wargames conducted by the Joint Experimentation Directorate within FDD and they provided an opportunity to immerse the participants deeply in the scenario. The wargames generated a concept of operations and an ORBAT ${ }^{1}$ for each LOO.

Project Global Endeavour (GE) have an established reputation for delivering workgroup demand analysis for deployment of ADF personnel on operations. They have developed a suite of models to help define what personnel are required to support an operation, going down to the level of specific qualifications. The models address the initial deployment as well as personnel required for both subsequent rotations and replenishing forces in the case of casualties. After establishing the personnel demand, the GE models compare that demand with the available supply of qualified personnel, which is obtained from Defence One personnel data. Their products have been used by Headquarters Joint Operations Command, Joint Capabilities Group and the Australian Army to inform operational and workforce planning.

As part of the analysis to support DCAP2020-21, GE observed the wargames and interviewed participants to establish the operational demand for workforce to achieve the Theatre Problem. This required some expansion of the existing models to include capabilities that are anticipated for 2029 but not part of the current force-inbeing. GE then compared the anticipated demand with the supply based on the 2021 workforce data, as illustrated in Table 1. Whilst the 2021 data does not show the growth that is planned for specific areas, this comparison still gives powerful insight into workforce challenges for Defence if facing this scale of operation. There are still gaps in the GE analysis; for example, the joint enabling functions, such as essential ICT functions, are not well covered by this modelling.

The GE operational demand analysis provides insight into the significance of specific workforce gaps that have been identified and is a powerful tool to inform decisions about which areas to allocate additional personnel in the planned growth. This modelling is the most detailed of all the workforce analysis undertaken to support DCAP2020-21.

Table 1. Illustration of the GE comparison of workforce demand against expected supply

\begin{tabular}{|c|c|c|c|c|c|c|c|}
\hline \multirow{2}{*}{$\begin{array}{l}\text { Person } \\
\text { Type }\end{array}$} & \multicolumn{4}{|c|}{$\begin{array}{c}\text { Demand } \\
\text { (Requirement to meet Theatre Problem) }\end{array}$} & \multicolumn{3}{|c|}{$\begin{array}{c}\text { Supply from } 2021 \text { Workforce } \\
\text { (Existing, well-understood data) }\end{array}$} \\
\hline & LOO1 & $\mathrm{LOO} 2$ & LOO3 & Peak & $\begin{array}{l}\text { Number } \\
\text { available }\end{array}$ & Initial deployment & $\begin{array}{c}\text { Sustain for } \\
\text { duration }\end{array}$ \\
\hline$\ldots$ & 14 & 2 & 0 & 14 & 10 & & \\
\hline$\ldots$. & 2 & 7 & 9 & 16 & $\ldots$ & & \\
\hline$\ldots \ldots$ & 20 & 10 & 15 & 25 & & & \\
\hline$\ldots$. & $\ldots$ & $\ldots$ & $\ldots$ & $\ldots$ & $\ldots$ & & \\
\hline & & & & & & & \\
\hline
\end{tabular}

\footnotetext{
${ }^{1}$ ORBAT stands for Order of Battle and refers to the list of equipment and resources available to a military commander in an operation.
} 


\subsection{Bayesian Reasoning Value Model: Capability Impact of Additional Workforce}

One way to model the interconnectedness of Defence capabilities is through Bayesian network modelling. A Bayesian model was developed to capture how the constituent parts of the Defence contribute to the whole in a specific warfighting context (Nguyen et al. 2021). Figure 1 shows conceptually the structure of the Bayesian Reasoning Value Model (BRVM) that was developed. At the core of the model are force packages (FP), which are sets of Defence Elements (for example, a Joint Strike Fighter aircraft or an Air Warfare Destroyer ship) that typically operate together. The middle layer of the model are Operational Effects $(\mathrm{OE})$, which are specific effects that need to be delivered to achieve the mission. OEs are described by the set of tasks that must be achieved. Several operational effects work together to achieve a decisive condition (DC), which is similar to a set of interrelated decisive points and can be considered as an overall mission for the Line of Operation. Achieving success in the decisive conditions contributes to success in the overall Theatre Problem at the top. Within the model, each of these nodes has a probability of success that is determined by the state of its parent nodes, i.e. the things it depends on.

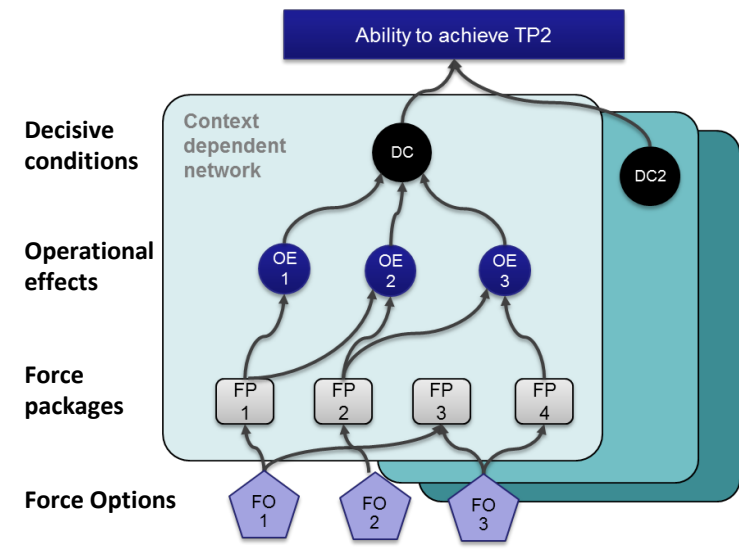

Figure 1. Conceptual structure of the Bayesian Reasoning Value Model
The Force Packages are actually represented by two nodes: the performance and the availability of the force package. Availability captures both the capacity and sustainability of the force package elements and can be summarised as "Is the force package available to do the mission?" Performance assumes that the force package is available and asks "Can the force package get the job done?" Force package performance and availability determine the ability of Defence to deliver the operational effects to achieve the decisive conditions and ultimately to win the war described by the Theatre Problem.

At the bottom of Figure 1 are the Force Options, which are possible investments or divestments in military capability that can impact the force package performance and availability.

The BRVM was constructed by DSTG based on the findings from the operational wargames described in section 3.1. One big advantage of this model is that the impact from one change to the force can be considered across multiple causal chains, so that the different parts of the operation are not stove-piped and the complex interdependencies can be explored.

The overall purpose of the BRVM is to help us understand a very complex and interconnected system. It also allows us to quantify the benefit from proposed investments in terms of the improvement in the overall probability of succeeding in the Theatre Problem. In DCAP 2020-21, the BRVM was used to generate Capability Value Scores for proposed additional workforce bids, based on its impact on force package performance and availability and subsequent contribution to the overall mission of the Theatre Problem.

\subsection{Workforce to Support the Defence Business Enterprise}

The above analysis methods focus on understanding the operational or warfighting capabilities of Defence. However, this analysis of future workforce needs must also address the enabling and supporting functions within the Defence organisation.

The DCAP 2020-21 cycle used the traditional scenario-based approach for the 'operational' force options and direct assessment of options using multi-criteria decision analysis for the 'enterprise' or business force options. The 'operational' force options were those that could be tested through the operational scenario and included some enabling functions such as logistics, intelligence and information technology infrastructure. The value or benefit expected from investment in the operational force options was assessed primarily using the BRVM described in section 3.2.

The remainder were 'enterprise' force options, which were proposed investments in the business functions of Defence and other enabler systems that are too far removed from delivering military effects to be meaningfully considered against a warfighting scenario. These enterprise functions included capability acquisition, facility management, finance, military strategy, force design, science and technology, and the engagement functions with the rest of the government. 
DSTG applied a multi-criteria decision analysis method to assess the enterprise force options in a series of workshops. Representatives from every Group and Service of Defence generated a narrative of how the Force Option mitigates risk to the Defence enterprise, including against targets in the Defence Corporate Plan. To score each group's options, the participants used these narratives to perform pairwise comparisons using the Best Worst Method (Rezaei 2015), where every option was rated on a Likert scale against what they chose as the best and the worst option. The option lists from the Groups and Services were stitched together in facilitated sessions, using a visual tool that allowed linear scaling and translation to place them on a common scale. Senior decision makers accepted the enterprise scores as a fair representation of future Defence requirements (Boyce et al. 2021).

These enterprise option scores were stitched together with the operational scores to create a single set of quantitative value assessments. By distinguishing between these operational and enterprise subsets of Defence and holistically assessing the requirements for both, Defence has been able to elicit an all-encompassing view of its future workforce requirements.

\subsection{Boxarr Workforce Iceberg Model: Attributing Existing Workforce to Defence Capability}

Another lens for understanding Defence workforce needs was to examine holistically how the current Defence workforce supports capability, including all of the enterprise, business and support functions within the Department of Defence. For this work, FDD developed a model within Boxarr, which is a commercial modelling package aimed at mapping business processes (About Boxarr 2021). The Boxarr model again uses force packages as the basic partitioning scheme for Defence capability.

Figure 2 shows the basic architecture that was used for this model. Each force package was represented by four layers in the workforce pipeline iceberg ${ }^{2}$, which is the training and readiness pipeline required to prepare personnel for deployment. At the top, 'deployed' means those personnel deployed on operations as part of a Joint Task Force, although in this model this peak actually represents all personnel who are fully trained and fulfilling their roles in operations, whether overseas or from an Australian base. The lower layers represent the personnel required to generate and support the deployed force. Personnel in Operations Generation or 'OPGEN' are those preparing to deploy in a specific operation. 'FORGEN' or Force Generation refers to the larger pool of available workforce, often described as being within the Raise, Train and Sustain (RTS) process. The terms OPGEN and FORGEN originate as two lines of effort in generating Army forces: "FORGEN itself, in which it is preparing for 'a war,' and Operations Generation, which prepares forces for a specific war or 'the war" (Butler et al. 2018 , p. 8). Underpinning the RTS process is the recruitment and ab initio training of new recruits, which sits at the bottom of the iceberg.

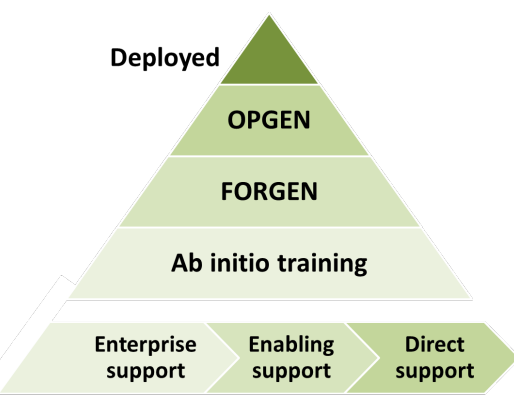

Figure 2. Workforce Iceberg Model

Many of the enabling and supporting functions sit outside of this workforce pipeline construct; for example, deeper-level maintenance of submarines is conducted by a completely separate workforce from the training and preparation of submariners. These supporting functions were described as being in 'Direct Support', 'Enabling Support' or 'Enterprise Support'. The goal of the Boxarr workforce iceberg model is for every part of Defence to be mapped onto a force package, either contributing to a layer in the iceberg or as a part of the supporting workforce. For areas that contribute across capabilities, they could either be weighted towards one aspect or spread evenly across them all.

The Boxarr model helped force designers to understand how every part of Defence supports the warfighting capability, and to expose the fragility of the workforce pipeline for some areas. When this information is combined with information about the criticality of different force packages (for example, from the BRVM), it can provide insight on the benefit of investment or the cost of divestment in different areas across Defence.

There were a number of limitations of this model. Firstly, the interpretation of the personnel numbers in the source data was different for different areas due to inconsistent policies around "positions" compared to actual people. Different work categories such as military reservists, contractors and APS could not be distilled down to a common basis with permanent ADF. Secondly, there was insufficient time to unravel all the complexities

\footnotetext{
${ }^{2}$ Called an iceberg because most of the people needed to deliver the deployed force are hidden below the surface.
} 
for how some areas support other areas. Finally, the force package construct maps more readily to organisational structures in some areas than others. For example, the OPGEN/FORGEN construct was more applicable to Army and Navy models of deployment, whereas the Air Force has a modularised approach to workforce deployments and their utility of key assets. Overall, this model is more reliable for some parts of Defence than for others and should be used with caution at this stage of its maturity.

\subsection{Workforce Growth Achievability and Supply Modelling}

The above analysis methods have primarily focussed on understanding future workforce needs and where the planned workforce might fall short. A good workforce plan must also be informed by realistic estimations of achievable growth rates. Achievable workforce growth is a function of recruitment, retention and training, and all of these are functions of time. Furthermore, the cost of recruitment, training and absorption ${ }^{3}$ is key to determining achievability when balanced against other funding requirements. The workforce supply modelling undertaken by DPG uses Markov chain Monte Carlo simulations to develop a picture of how a component of the workforce might realistically be able to grow and when specific trained workforces will be realised in order to meet capability requirements. The use of Markov chains for military workforce simulation is well established (Zais and Zhang 2015) and the models developed for DCAP 2020-21 built on previous analysis from within DPG (Wang 2005).

Analysis of the historical data indicates that duration of service is a significant predictor of separation behaviours, likely due to training periods, minimum service obligations and retention benefits (Cunha et al. 2015). Predictions also depend on key demographics such as Service (i.e. Navy, Army and Air Force), military rank, and occupation or employment category. The ADF military workforce has over 200 employment categories and twenty specific military ranks. Whilst many of these occupations have specific behaviours, this analysis aggregated them into eight macro workforce segments: combat and security, logistics, engineering, maintenance and construction, enterprise and command support, intelligence, communications and cyber, health and aviation. The rank structure was simplified to Officer and Other ranks. A total of 48 models were developed ( 3 services $\times 2$ rank groups $\times 1$ segments) and each model contained two Markov chains to capture the transition behaviours each year in either 'training force' or 'trained force'. Figure 3 shows a basic process for how individuals might move through the training pipeline on the way to becoming fully qualified and trained personnel. A total demand profile for each model was informed by existing structures and from the outputs of the workforce

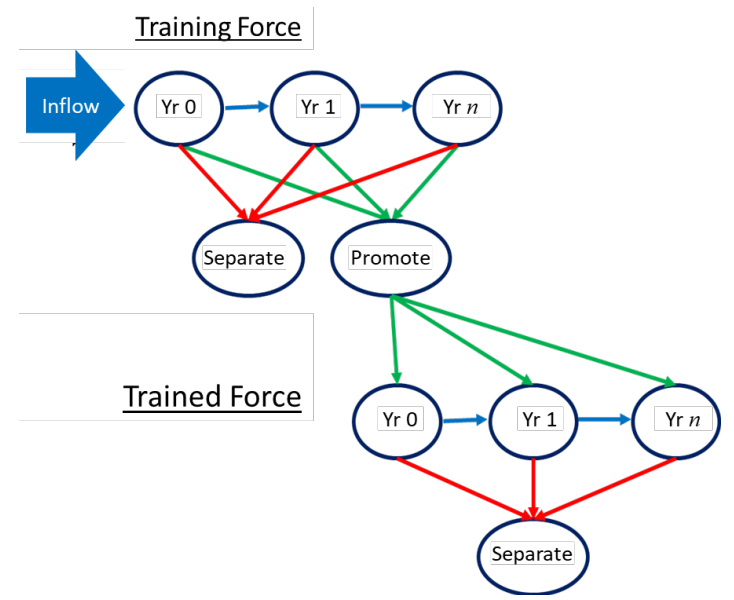

Figure 3. Markov chain simulation is used to model how inflows of new recruits translate to trained personnel over time demand modelling described in this paper.

By manipulating the inflow for each time step and minimising the gap between supply and demand through the use of a bespoke evolutionary generation and target-seeking optimisation algorithm, the model was able to provide workforce planners with indicative inflows and headcounts for training and trained force. This was further extrapolated to provide details for specific training schools and locations. This information was then fed back into the various workforce planning and cost activities to inform overall achievability in terms of inflow, training requirements and additional costs such as significant new infrastructure requirements.

Overall, workforce achievability is not a hard constraint on workforce growth, but a way to inform what it might cost to on-board personnel at a particular rate. The costs can arise from scaling up recruitment and training centres, as well as accommodation and other infrastructure.

\footnotetext{
${ }^{3}$ Absorption refers to providing the physical workspaces and housing needed to ensure the workforce can effectively perform their duties. In a military setting, this can also include community infrastructure and services needed to support military families.
} 


\section{DISCUSSION}

This campaign of analysis to understand future Defence workforce requirements created new connections among different analysis methods. This strengthened each individual approach and enabled the design of coherent, achievable and targeted workforce growth options for Defence. The operational workforce demand modelling by GE has potential to inform a range of future analysis, including to test assumptions about the Defence capacity for concurrent operations and to inform force package availability estimates in the BRVM. One limitation that is common to both the operational workforce demand and the BRVM is that they rely on the same scenario as the exemplar for future demands on Defence and were built from data collected at the same operational wargames. Whilst the Theatre Problem used is a thorough and complex test of Defence capability, it still stresses some capabilities more than others. Any user of these models must be cognisant that results are most relevant to that specific set of vignettes.

The workforce growth modelling examined the ability to recruit, train and retain the required workforce within eight workforce segments. By aggregating the wide variety of occupations, ranks and locations into these broad groups some detail is lost and further analysis is needed to ensure that the growth plan for specific critical occupations is still achievable.

\section{CONCLUSION}

A campaign of analysis has been conducted to help Australian Defence workforce planners to understand future requirements and design coherent, achievable and targeted workforce growth options for the ADF. Five complementary analytical approaches were employed, drawing on expertise in DPG, DSTG, and the FDD of the ADF Headquarters. Based on a series of wargames, operational demand modelling provided a detailed estimation of the number and types of people Defence will need to meet a future warfighting scenario. A Bayesian network model captured how the constituent parts of Defence contribute in a warfighting context, and was used to quantify the expected benefit from proposed workforce investments. The supporting and enabling functions of Defence were considered through both a prioritisation of proposed investments and mapping how the entire organisation supports capability. Workforce growth and supply modelling provided realistic estimations of achievable growth rates.

The FSP20 identified that the Defence workforce needs to grow to support a larger number of more-complex capabilities, including to build capacity in cyber, intelligence, electronic warfare and space. The analytical campaign described in this paper has provided much-needed detail to underpin how Defence will reshape and reskill its workforce in response to these emerging requirements.

\section{ACKNOWLEDGEMENTS}

This paper represents the work of several large teams to develop the underpinning models; in some cases developed over many years. The authors would like to thank Joint Experimentation Directorate for the conduct of the operational wargames that generated essential data to create the BRVM and GE models. Thanks also to the many participants and subject matter experts who offered their expertise to inform and build the models for this analysis.

\section{REFERENCES}

About Boxarr. 2021, viewed 4 August 2021, https://www.boxarr.com/about-us.

Boyce, J, et al. 2021, Consideration of enabling and enterprise functions within Defence force design, In preparation for: 24th International Congress on Modelling and Simulation, Sydney.

Butler, DM, et al. 2018, Comprehensive analysis of strategic force generation challenges in the Australian Army. RAND Corporation, Santa Monica, CA, USA, RR-2382-AUS.

Cunha, JM, et al. 2015, The retention effects of high years of service cliff-vesting pension plans. Economics Letters 126, 6-9.

Department of Defence 2020, 2020 Force Structure Plan. Canberra, Australia.

Nguyen, M-T, et al. 2021, An evaluation model and tool for Defence capability options, In preparation for: 24th International Congress on Modelling and Simulation, Sydney.

Rezaei, J 2015, Best-worst multi-criteria decision-making method. Omega, (53), 49-57.

Wang, J 2005, A review of operations research applications in workforce planning and potential modelling of military training. Defence Science and Technology Organisation, DSTO-TR-1688.

Zais, M \& Zhang, D 2015, A Markov chain model of military personnel dynamics. International Journal of Production Research 54, (6), 1863-85. 